Park et al. ${ }^{2}$ evaluated their technology in a transgenic mouse line designed to express ChR2 under the control of the promoter for the sensory neuron-specific gene Advillin (Advillin-ChR2). When implanted on top of the sciatic nerve or over the spinal cord, their wireless flexible optical stimulators drive unconditioned place aversion due to the triggered pain response. The authors also found similar effects in mice expressing ChR2 in nociceptors under the TrpV1 promoter (TrpV1-ChR2).

Both teams have made strides toward simplicity and affordability to facilitate adoption of their technologies. Although the approach by Montgomery et al. ${ }^{3}$ permits straightforward fabrication of implantable receiver-LED assemblies, it relies on the evanescent coupling of the $\mathrm{RF}$ field into the body of the animal. As a result, it requires close proximity of the subject to the RF cavity, restricting the behavioral paradigms that can be applied to the two-dimensional format. In contrast, the strategy pursued by Park et $a l{ }^{2}$, which uses direct free-space RF transmission, could be generalized to three-dimensional environments, albeit at the expense of highly specialized fabrication methods for the flexible antenna. The foreign body response remains a major challenge for implantable neuromodulators, and both teams have attempted to address it either through extreme miniaturization of a rigid device ${ }^{3}$ or through matching of the mechanical properties of the device to the tissue $^{2}$. Although both teams provided encouraging preliminary data for their technologies, quantitative analyses are needed to unequivocally confirm the biocompatibility and reliability of the devices in long-term experiments.

New wireless optogenetic tools promise several advantages over their tethered or head-mounted predecessors. They will permit optogenetic experiments on animals in sophisticated naturalistic environments. They should also be useful for studies of excitable cells outside the brain, such as those in the enteric nervous system. For example, the role of neural interactions between the brain and the gut could be addressed optically in a context of inflammatory conditions as well as metabolic diseases. Finally, integrating these wireless implantable devices with neural recording or biomarker-sensing capabilities, while challenging, could in principle enable closed-loop stimulation paradigms. Although these combinatorial approaches remain elusive, in the future they should make possible highly parallelized and automated behavioral studies to monitor long-term progression and identify future therapeutic interventions for psychiatric and neurological conditions such as schizophrenia or Parkinson's disease.

COMPETING FINANCIAL INTERESTS

The author declares no competing financial interests.
1. Boyden, E.S., Zhang, F, Bamberg, E., Nagel, G. \& Deisseroth, K. Nat. Neurosci. 8, 1263-1268 (2005).

2. Park, S.l. et al. Nat. Biotechnol. 33, 1280-1286 (2015).

3. Montgomery, K.L. et al. Nat. Methods 12, 969-974 (2015).

4. Nussinovitch, U. \& Gepstein, L. Nat. Biotechnol. 33 750-754 (2015).

5. Bruegmann, T. et al. Nat. Commun. 6, 7153 (2015).
6. Magown, P., Shettar, B., Zhang, Y. \& Rafuse, V.F. Nat. Commun. 6, 8506 (2015)

7. Yizhar, O., Fenno, L.E., Davidson, T.J., Mogri, M. \& Deisseroth, K. Neuron 71, 9-34 (2011).

8. Lee, S.T. et al. IEEE Trans. Neural Syst. Rehabil. Eng. 23, 655-664 (2015).

9. Kim, T.l. et al. Science 340, 211-216 (2013)

10. Jeong, J.W. et al. Cell 162, 662-674 (2015).

11. Yeh, A.J. et al. Appl. Phys. Lett. 103, 163701 (2013).

\title{
A systems approach to HIV-1 vaccines
}

\section{Tianlei Ying, Ponraj Prabakaran \& Dimiter S Dimitrov \\ Understanding the full breadth of immune responses that protect against HIV-1 may speed vaccine development.}

More than three decades after the first reports of AIDS, we still lack an effective HIV-1 vaccine. Recent clinical trial results have hinted at the complexity of the immune responses that could protect against HIV-1. Not only broadly neutralizing antibodies but also immune functions mediated by the antibody Fc (fragmentcrystallizable) region seem to be protective. A comprehensive description of anti-HIV-1 immunity could aid the design and testing of improved vaccines. In a recent paper in Cell, Chung et al. ${ }^{1}$ studied the antibody profiles of participants in four vaccine clinical trials in unprecedented detail and used a systems analysis to better define immune 'correlates of protection' (Fig. 1 and Table 1).

Vaccine developers have traditionally focused on discovering agents that elicit high titers of neutralizing antibodies against the pathogen. For HIV-1, considerable evidence suggests that neutralizing antibodies are indeed protective. In nonhuman primate models of HIV-1 infection, passive administration of neutralizing antibodies has achieved complete protection ${ }^{2}$. A recent first-in-man trial showed that a broadly neutralizing antibody-an antibody capable of neutralizing most viral strains — can significantly reduce viral load in HIV-1-infected individuals ${ }^{3}$. However, monoclonal antibodies are expensive, and they are typically administered therapeutically, after infection, rather

Tianlei Ying is at the Key Laboratory of Medical Molecular Virology, Ministries of Education and Health, School of Basic Medical Sciences, Fudan University, Shanghai, China. Ponraj Prabakaran is at the Intrexon Corporation, Germantown, USA. Dimiter Dimitrov is at the Center for Cancer Research, National Cancer Institute, National Institutes of Health, USA. e-mail:dimiter.dimitrov@nih.gov than prophylactically. Therefore, the quest for an efficacious AIDS vaccine continues.

One promising strategy, initiated by our studies of germline precursors of broadly neutralizing antibodies ${ }^{4}$, is to develop immunogens that stimulate maturation of antibody precursors ${ }^{5}$. Substantial effort along these lines is ongoing ${ }^{6}$, but the complexity of antibody maturation pathways remains a challenge. Another approach is to look beyond neutralizing antibody activity to other immune correlates of protection. Both neutralizing and non-neutralizing antibodies against HIV-1 could act through antibody Fc-related functions, such as antibody-dependent cellular cytotoxicity (ADCC), antibody-dependent cellular phagocytosis (ADCP) and antibodydependent complement deposition (ADCD).

Recent analyses of HIV-1 vaccine trials seem to support a role of Fc-dependent processes. Of particular interest is the RV144 trial, a randomized, double-blind efficacy trial of a prime-boost HIV-1 vaccine that showed a modest $(31.2 \%)$ reduction in the risk of infection at 42 months ${ }^{7}$. Interestingly, broadly neutralizing antibodies were not detected in any participant analyzed. Subsequent studies of samples from this trial found that antibodies (IgG) specific to a particular region (V1V2) of the HIV-1 envelope glycoprotein (Env) correlated inversely, and other antibodies (IgA) directly, with infection rates among those who were vaccinated ${ }^{8}$, and that IgG3 was important for protection ${ }^{9}$. In vaccinees with low IgA levels, ADCC, IgG avidity and neutralizing activity were inversely correlated with infection ${ }^{7}$, indicating that ADCC could contribute to protection in some vaccinees ${ }^{8}$.

In their 2014 study, Chung et al. ${ }^{10}$ sought to further dissect the role of Fc-mediated 


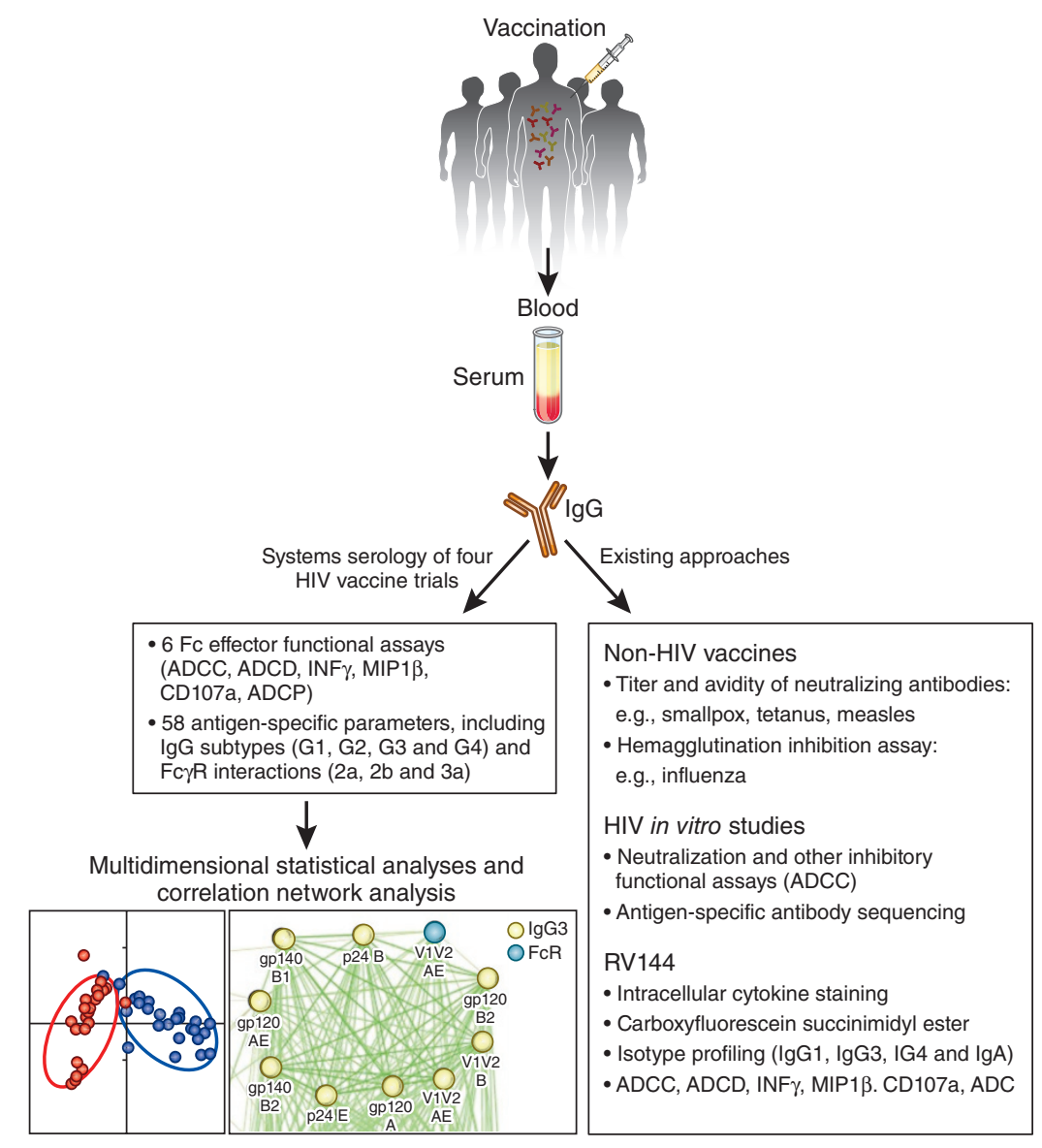

Figure 1 An integrated serological profiling approach reported by Chung et al. ${ }^{1}$ for identification of unique humoral Fc-related vaccine signatures (left) compared to other approaches (right). Chung et al. ${ }^{1}$ purified IgG from participants in four different HIV-1 vaccine trials (RV144, VAX003, HVTN204 and IPCAVD001). Six Fc effector functions and 58 biophysical characteristics were evaluated, including binding to Fc $\gamma$ receptors and relative abundances of different antigen-specific antibodies in 120 samples. All 64 parameters were subjected to statistical analyses, including hierarchical clustering, multidimensional combined feature selection method, partial least-squares discriminant analysis and correlation network analysis, to identify unique signatures and differences that may be related to protection. Previous methods typically included many of these parameters but were rarely based on extensive evaluation of coordinated Fc functions.

responses in the RV144 trial. They compared antibody profiles in plasma samples from 20 placebo recipients and 80 vaccinees with those from 10 placebo recipients and 50 vaccinees in the ineffective VAX003 trial. They showed that in the RV144 trial, highly functional IgG3 was elicited, whereas in the VAX003 trial the IgG3 response was negatively affected by IgG4, and also that IgG1 and IgG3 antibodies targeting the crown of the HIV-1 Env V2 were elicited only in the RV144 trial. These findings are important because the differences between IgG3 and IgG4, which are associated with coordinated responses targeting V2 epitopes, may underlie the observed modest efficacy in the RV144 trial. Interestingly, depletion of IgG3 significantly reduced both ADCP and ADCC in vitro, indicating that $\mathrm{Fc}$ effector functions could be important in vivo.

In the Cell paper just published, Chung et al. ${ }^{1}$ expanded their previous study ${ }^{10}$ to include two additional trials that failed to show protection (HVTN204 and IPCAVD001) (Table 1). They measured multiple parameters using blood samples from all four trials: Fc-related functions (ADCC, ADCP, ADCD and three antibody-dependent natural killer (NK) cell functions-secretion of IFN $\gamma$ and of MIP1 $\beta$ and degranulation) together with biophysical parameters (58 relative concentrations, including bulk IgG, IgG1, IgG2, IgG3 and IgG4; binding to gp140, gp70, p24, gp120 and gp41 from clades $\mathrm{B}, \mathrm{AE}$ and $\mathrm{A}$; ten relative concentrations of IgA included only in the analysis of the RV144 trial; and binding to Fc $\gamma$ R2a, Fc $\gamma R 2 b$ and Fc $\gamma R 23 b)$. The analysis of such a large number of parameters from four different clinical trials was enabled by the use of much more sophisticated statistical analyses than the simple correlation statistics used in previous studies. This integrative approach should help to identify new parameters, and relationships between them, that could not only identify correlates of protection but also be useful in elucidating mechanisms of antibody elicitation by vaccine immunogens.

The authors initially used unsupervised hierarchical clustering to group vaccine regimens by the type of immunogen involved. Although this method revealed the dominance of immunogen type in eliciting distinct profiles, it was not possible to dissect specific features responsible for the separation of clusters. To obtain enhanced resolution of vaccine signatures, the authors used classification and discrimination methods as well as a correlation network analysis. The RV144 and VAX003 profiles were clearly separated through the use of as few as seven features. The analysis confirmed the previous finding of elevated IgG3 in the RV144 trial and dominance of IgG4 in the VAX003 trial. It also identified features that might be related to the lack of protection in VAX003, including elevated gp140-specific antibodies, NK cell degranulation and chemokine secretion, indicating that induction of those responses might not be important for an efficacious HIV-1 vaccine.

The authors also found that clear separation of the antibody signatures of the four vaccine trials could be achieved by analysis of just 15 of the 64 measured parameters. One interesting result was that the antigen itself, rather than the vector or immunization regimen, significantly affected the type of antibody profile elicited. The correlation networks for the four trials proved to be very different; this part of the analysis provides unique information about relationships between features that contribute to differences among the trials. Identification of networks with specific feature-function relationships could help to elucidate mechanisms underlying protection. For example, in the RV144 trial, the total IgG binding to $\mathrm{V} 1 \mathrm{~V} 2$ regions from the Env clades A and E was directly tethered to both ADCP and ADCC, suggesting that this specific response to V1V2 could drive the killing of infected cells through ADCP and ADCC.

The 'systems serology' analysis presented by Chung et al. ${ }^{1}$ suggests that IgG3 in the RV144 could either be a surrogate of effective responses or contribute to an effective response through combination with multiple other antibody features, particularly IgG1. Interestingly, they also found that IgA was an antagonist of all of the IgGs specific to V1V2 except IgG3, and therefore IgA could be a marker of less effective immune responses. Finally, their results also indicated that ADCP could be crucial in combination with Fc-related functions for protection from the transmission of HIV-1 through mucosal barriers.

Although the approach of Chung et al. ${ }^{1}$ promises to enable exquisitely detailed analyses 
Table 1 The four trials analyzed by Chung et al. ${ }^{1}$

\begin{tabular}{|c|c|c|c|c|c|c|}
\hline Trial & Immunogen & Vector & $\begin{array}{l}\text { Immunogen } \\
\text { administration }\end{array}$ & Participants & Start date & Outcome \\
\hline \multirow[t]{2}{*}{ RV144 } & Prime:gag-pr-gp41-gp120 & Canarypox & $0,4,12,24$ & \multirow[t]{2}{*}{16,403} & \multirow[t]{2}{*}{ October 2003} & \multirow{2}{*}{$\begin{array}{l}\text { Some }(31.2 \%) \\
\text { efficacy }\end{array}$} \\
\hline & Boost: gp120 (clade B,E) & Subunit proteins & 12,24 weeks & & & \\
\hline VAX003 & gp120 (clade B, E) & Subunit proteins & $0,1,6,12,18,24,30$ months & 2,500 & March 1999 & No efficacy \\
\hline \multirow[t]{2}{*}{ HVTN204 } & Prime: Env (clades $A, B, C$ ), gagB, polB, nefB & DNA & $0,1,2$ months & 480 & September 2005 & No efficacy \\
\hline & Boost:: Env (clades $A, B, C$ ), gagB, polB, & rAd5 & 6 months & & & \\
\hline IPCAVD 001 & Env clade $A$ & rAd26 & 0 weeks & 60 & February 2008 & No efficacy \\
\hline
\end{tabular}

Serum samples (30 vaccinees for each trial) were taken 2 weeks after the last immunization.

of Fc-related vaccine signatures, there are practical and methodological concerns that will have to be taken into consideration when undertaking such analyses. For example, the assays used for the Fc effector functions are not easy to standardize across laboratories. Future studies would benefit from the use of blinded samples. It would also be of interest to test samples from lymphoid tissues, the major site of HIV-1 replication, in addition to blood samples. The results of systems analyses depend on the breadth and depth of the training data set and often cannot be generalized. Thus, the authors' conclusions may not hold for other immunogens or viruses. Finally, in the RV144 trial, only very limited protection was achieved and less than $1 \%$ of all participants were infected, so the significance of any identified correlate of protection remains to be determined.

Nevertheless, a systems serology approach could be readily applied to other trials and expanded to include additional parameters, such as sequence analysis of antibodyomes ${ }^{4}$ and molecular signatures of vaccines. Such studies might also be designed to address the important question of how to predict the efficacy of a novel vaccine based on in vitro studies or animal models. Finding the immunological correlates of vaccine protection for HIV-1 infection by considering qualitative and quantitative immune responses could help guide us to the next generation of vaccines ${ }^{11}$.

\section{COMPETING FINANCIAL INTERESTS}

The authors declare competing financial interests: details are available in the online version of the paper.

1. Chung, A.W. et al. Cell 163, 988-998 (2015).

2. Shibata, R. et al. Nat. Med. 5, 204-210 (1999).

3. Caskey, M. et al. Nature 522, 487-491 (2015).

4. Xiao, X. et al. Biochem. Biophys. Res. Commun. 390 404-409 (2009).

5. Dimitrov, D.S. MAbs 2, 347-356 (2010).

6. Haynes, B.F., Kelsoe, G., Harrison, S.C. \& Kepler, T.B. Nat. Biotechnol. 30, 423-433 (2012).

7. Rerks-Ngarm, S. et al.; MOPH-TAVEG Investigators. N. Engl. J. Med. 361, 2209-2220 (2009).

8. Haynes, B.F. et al. N. Engl. J. Med. 366, 1275-1286 (2012).

9. Yates, N.L. et al. Sci. Transl. Med. 6, 228ra39 (2014).

10. Chung, A.W.E. et al. Sci. Transl. Med. 6, 228ra38 (2014).

11. Corey, L. et al. Sci. Transl. Med. 7, $310 r v 7$ (2015).

\section{Getting rid of PERVs}

CRISPR-Cas technology has been used in a broad range of applications, including multiplexing. Up to five genes have been targeted in the same cell ${ }^{1}$, but whether this number could be extended by much was not clear. In a new demonstration of the capabilities of the CRISPR-Cas system, George Church, Luhan Yang and colleagues recently reported simultaneously editing 62 related genes in pig cells ${ }^{2}$.

Engineering the genome of pigs is not a new idea. A strong incentive for altering the genetic makeup of these animals is to make them more suitable as donors for xenotransplantation. Pig organs are roughly the same size as those of humans and share similar vascular architecture. Tens of thousands of individuals could receive life-saving transplants every year if the remaining hurdles in xenotransplantation were overcome. One such challenge is the potential transmission of porcine endogenous retroviruses (PERVs) to humans.

Aiming to inactivate PERVs, Church, Yang and colleagues began by determining how many PERV elements are present in the genome of pig cells, and identified 62. Then, they designed two Cas9 guide RNAs that would target the pol gene, an essential and conserved PERV gene, in all 62 PERVs. They expressed the guide RNAs and Cas9 in PK15 cells, a pig kidney epithelial cell line, and sequenced clones to determine the extent of editing that had occurred. They found a small number of clones that had up to $100 \%$ disruption of the pol gene, along with evidence suggesting that this high efficiency was due to a mechanism in which previously edited pol genes were used as templates to disrupt wild-type pol genes. Elucidating the intricacies of this mechanism may uncover interesting biology and further potential applications of the approach.

"This illustrates the power of the CRISPR technology," says Randall Prather, of the University of Missouri. "A few years ago we wouldn't have thought of doing this because inactivating 62 genes, or even a family of 10 genes, would have been quite complicated with traditional homologous recombination."

Editing 62 genes, even if closely related, is certainly a feat, and the engineered cells showed a 1,000-fold reduction in PERV transmission to human cells. That said, whether PERV transmission is likely to be a problem in xenotransplantation is not clear. Kevin Wells, also from the University of Missouri, says that PERV transmission was on the list of things to examine, but probably not at the top. "We don't know what the actual risk is," he says. Although there is evidence that PERV transmission can occur in vitro, "no one has observed transmission of PERVs in experiments where a nonhuman primate received a pig organ," says Wells. In addition, alternative approaches could be used, such as employing pig lines that do not produce human-tropic, replication-competent PERVs ${ }^{3}$.

Organ rejection continues to be the major hurdle in xenotransplantation. Although acute organ rejection has been addressed, immune rejection is still a considerable problem. On that front, Wells points out that CRISPR-Cas9 genome editing could be used to

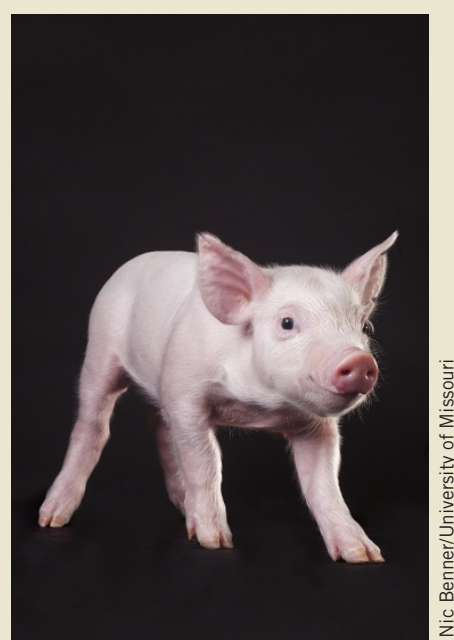

The 62 endogenous porcine retrovirus genes have been inactivated in pig cells. Are piglets next?

target pig genes encoding antigenic carbohydrates or proteins. Inactivation of porcine circulating proteins that don't interact well with their human counterparts could also help efforts to make xenotransplantation a reality.

\section{Irene Jarchum, Associate Editor}

1. Wang, H. et al. Cell 153 910-918 (2013).

2. Yang, L. et al. Science 350, 1101-1104 (2015).

3. Oldmixon, B.A. et al. J. Virol. 76, 3045-3048 (2002). 